\title{
Thermodynamic Bounds on Precision in Ballistic Multiterminal Transport
}

\author{
Kay Brandner, ${ }^{1}$ Taro Hanazato, ${ }^{2}$ and Keiji Saito ${ }^{2}$ \\ ${ }^{1}$ Department of Applied Physics, Aalto University, 00076 Aalto, Finland \\ ${ }^{2}$ Department of Physics, Keio University, 3-14-1 Hiyoshi, Yokohama 223-8522, Japan
}

(Received 10 October 2017; revised manuscript received 28 December 2017; published 2 March 2018)

For classical ballistic transport in a multiterminal geometry, we derive a universal trade-off relation between total dissipation and the precision, at which particles are extracted from individual reservoirs. Remarkably, this bound becomes significantly weaker in the presence of a magnetic field breaking timereversal symmetry. By working out an explicit model for chiral transport enforced by a strong magnetic field, we show that our bounds are tight. Beyond the classical regime, we find that, in quantum systems far from equilibrium, the correlated exchange of particles makes it possible to exponentially reduce the thermodynamic cost of precision.

DOI: 10.1103/PhysRevLett.120.090601

Heisenberg's uncertainty principle is a paradigm example for the ubiquitous interplay between fluctuations and precision. It entails that the accuracy of simultaneous measurements of noncommuting observables is subject to a fundamental lower bound arising from intrinsic fluctuations in the underlying quantum states. Quite remarkably, the precision of nonequilibrium thermodynamic processes might be restricted through thermal fluctuations in a similar way: Barato and Seifert recently suggested that steady-state biomolecular process are subject to a universal trade-off between entropy production and dispersion in the generated output [1]. Since its discovery, this thermodynamic uncertainty relation has triggered significant research effort. A general proof based on methods from large-deviation theory was given by Gingrich et al. for Markov jump processes satisfying a local detailed balance condition [2,3]. Further developments include extensions to finite-time [4,5] and discretetime [6] processes, Brownian clocks [7], and systems obeying Langevin dynamics $[8,9]$.

In light of these results, the question arises whether a fundamental bound on the precision of thermodynamic processes can be derived from first principles. An ideal stage to investigate this problem is provided by ballistic conductors, that is, devices whose dimensions are smaller than the mean free path of transport carriers. In such systems, the transfer of particles is governed by reversible laws of motion, while all irreversible effects are relegated to external reservoirs, a mechanism also know as moderate damping $[10,11]$. This structural simplicity not only enables the use of physically transparent models, it also leads to a direct link between microdynamics and thermodynamic observables. Features such as the inertia of carriers or Lorentz-type forces, which are not covered by Markov jump processes in position space, are thereby naturally included. These advantages have made ballistic models an important source of insights on classical [12-15] and quantum [16-20] transport mechanisms. Here, we use this framework to derive a thermodynamic uncertainty relation

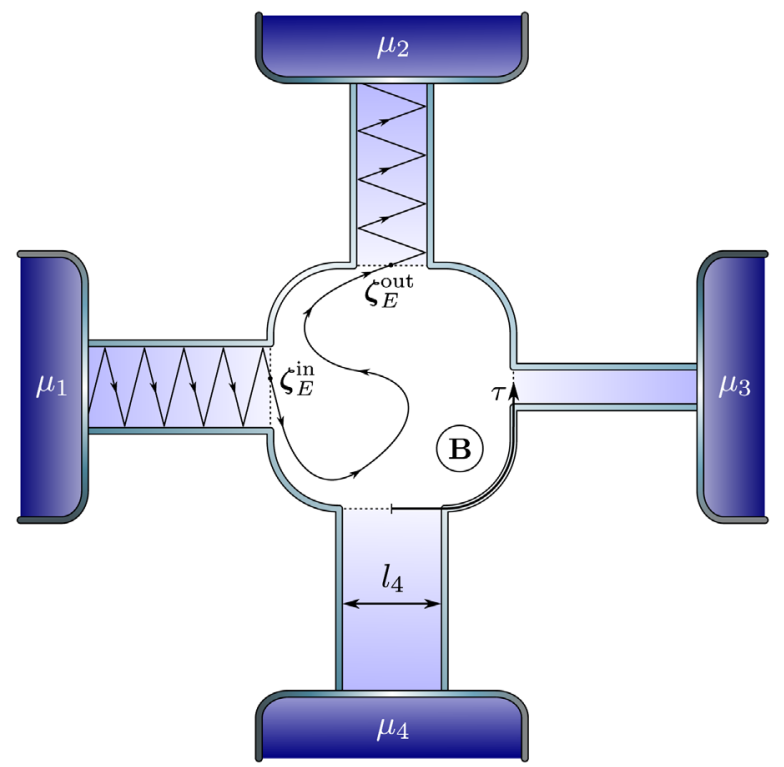

FIG. 1. Four-terminal setup as an example for multiterminal ballistic transport. A two-dimensional target is connected to four reservoirs denoted by their chemical potentials $\mu_{1}, \ldots, \mu_{4}$ via perfect leads of width $l_{1}, \ldots, l_{4}$. The solid line crossing the conductor shows the trajectory of a classical particle with energy $E$, which enters the target region at the point $\zeta_{E}^{\text {in }} \equiv\left(\tau^{\text {in }}, p_{\tau}^{\text {in }}\right)$ in the reduced phase space and leaves it at $\zeta_{E}^{\text {out }} \equiv\left(\tau^{\text {out }}, p_{\tau}^{\text {out }}\right)$ after being deflected by the target potential and the magnetic field $\mathbf{B}$. The coordinate $\tau$ parameterizes the boundary of the target region, and $p_{\tau}$ denotes the corresponding canonical momentum. Since the particle follows Hamiltonian laws of motion, the scattering map (2) is one to one. Because $\tau$ and $p_{\tau}$ are canonical variables, the Poincaré-Cartan theorem implies that this map is also volume preserving [21]. 
for classical ballistic transport, which can be traced back to elementary properties of Hamiltonian dynamics.

Scattering theory provides a powerful tool to describe ballistic transport in both the classical and the quantum regime. In this approach, the conductor is modeled as a target that is connected to $N$ perfect, effectively infinite leads. Each lead is attached to a reservoir with a fully transparent interface injecting thermalized, noninteracting particles. Once inside the conductor, the particles follow deterministic dynamics until they are absorbed again into one of the reservoirs (Fig. 1).

On the classical level, the current flowing in the lead $\alpha$ towards the target corresponds to a phase-space variable $J_{\alpha}\left[\xi_{t}\right]$, where the vector $\boldsymbol{\xi}_{t}$ contains the positions and momenta of all particles in the conductor at the time $t$. In the steady state, the mean value and fluctuations of this current are given, respectively, by

$J_{\alpha} \equiv \lim _{t \rightarrow \infty} \frac{1}{t} \int_{0}^{t} d t^{\prime}\left\langle J_{\alpha}\left[\boldsymbol{\xi}_{t^{\prime}}\right]\right\rangle$ and

$S_{\alpha} \equiv \lim _{t \rightarrow \infty} \frac{1}{t} \int_{0}^{t} d t^{\prime} \int_{0}^{t} d t^{\prime \prime}\left\langle\left(J_{\alpha}\left[\boldsymbol{\xi}_{t^{\prime}}\right]-J_{\alpha}\right)\left(J_{\alpha}\left[\boldsymbol{\xi}_{t^{\prime \prime}}\right]-J_{\alpha}\right)\right\rangle$,

where the average $\langle\bullet\rangle$ has to be taken over the ensemble of trajectories of injected particles [22].

Exploiting that the injected particles are statistically independent and noninteracting, the expressions (1) can be made more explicit. Focusing on two dimensions from here onwards, to this end, we decompose the trajectory of a single particle with energy $E$ into an incoming and an outgoing part connected by the scattering map:

$$
\mathcal{S}_{E, \mathbf{B}}: \zeta_{E}^{\text {in }} \mapsto \mathcal{S}_{E, \mathbf{B}}\left[\zeta_{E}^{\text {in }}\right]=\zeta_{E}^{\text {out }}
$$

The vectors $\zeta_{E}^{\text {in }}$ and $\zeta_{E}^{\text {out }}$ thereby contain the position and momentum of the particle as it enters and leaves the target region, and $\mathbf{B}$ denotes an external magnetic field applied to the target (Fig. 1). Using (2), we further introduce the dimensionless transmission coefficients

$$
\mathcal{T}_{E, \mathbf{B}}^{\alpha \beta}=\frac{1}{h} \int_{\beta} d \zeta_{E}^{\text {in }} \int_{\alpha} d \boldsymbol{\zeta}_{E} \delta\left[\mathcal{S}_{E, \mathbf{B}}\left[\boldsymbol{\zeta}_{E}^{\text {in }}\right]-\boldsymbol{\zeta}_{E}\right]
$$

where indices on integrals imply that the corresponding position variable runs only over the boundary between the target region and the respective lead and $h$ denotes Planck's constant. This definition allows us to compactly rewrite the mean currents and fluctuations (1) as [22]

$$
\begin{aligned}
J_{\alpha} & =\frac{1}{h} \int_{0}^{\infty} d E \sum_{\beta} \mathcal{T}_{E, \mathbf{B}}^{\alpha \beta}\left(u_{E}^{\alpha}-u_{E}^{\beta}\right) \quad \text { and } \\
S_{\alpha} & =\frac{1}{h} \int_{0}^{\infty} d E \sum_{\beta \neq \alpha} \mathcal{T}_{E, \mathbf{B}}^{\alpha \beta}\left(u_{E}^{\alpha}+u_{E}^{\beta}\right) .
\end{aligned}
$$

The chemical potentials $\mu_{\alpha}$ and temperature $T$ of the reservoirs enter these expressions via the MaxwellBoltzmann distributions

$$
u_{E}^{\alpha} \equiv \exp \left[-\left(E-\mu_{\alpha}\right) /\left(k_{B} T\right)\right]
$$

where $k_{B}$ denotes Boltzmann's constant. Note that the formulas (4) involve only single-particle quantities, while the original definitions (1) depend on the full phase-space vector $\xi_{t}$ of the many-particle system.

Maintaining the stationary currents $J_{\alpha}$ requires a strictly positive rate of entropy production [12,23,24]:

$\sigma \equiv k_{B} \sum_{\alpha} \mathcal{F}_{\alpha} J_{\alpha}=\frac{k_{B}}{h} \int_{0}^{\infty} d E \sum_{\alpha \beta} \mathcal{T}_{E, \mathbf{B}}^{\alpha \beta} \mathcal{F}_{\alpha}\left(u_{E}^{\alpha}-u_{E}^{\beta}\right)$,

which arises due to heat dissipation in the reservoirs. Thus, $\sigma$ can be regarded as the thermodynamic cost of the transport process, which is driven by the dimensionless thermodynamic forces $\mathcal{F}_{\alpha} \equiv\left(\mu_{\alpha}-\mu\right) /\left(k_{B} T\right)$ with $\mu$ denoting a reference chemical potential.

We will now show that this cost puts a universal lower bound on the relative uncertainty [1]

$$
\varepsilon_{\alpha} \equiv S_{\alpha} / J_{\alpha}^{2}
$$

of each individual current. To this end, we consider the quadratic form

$$
A_{\alpha} \equiv \sigma / k_{B}+2 \psi\left(J_{\alpha} x+S_{\alpha} x^{2} / 2\right),
$$

where $x, \psi \in \mathbb{R}$. Note that this object can be regarded as the second-order expansion of a modified cumulant-generating function describing the fluctuations of the current escaping the reservoir $\alpha$.

For systems without an external magnetic field, $A_{\alpha}$ can be written as

$$
\begin{aligned}
A_{\alpha}= & \sum_{\beta, \gamma \neq \alpha} \mathcal{V}^{\beta \gamma} \mathcal{D}_{\beta \gamma}\left(e^{\mathcal{D}_{\beta \gamma}}-1\right) / 2 \\
& +\sum_{\beta \neq \alpha} \mathcal{V}^{\alpha \beta}\left\{\left(\mathcal{D}_{\alpha \beta}+2 \psi x\right)\left(e^{\mathcal{D}_{\alpha \beta}}-1\right)+\psi x^{2}\left(e^{\mathcal{D}_{\alpha \beta}}+1\right)\right\}
\end{aligned}
$$

with $\quad \mathcal{V}^{\alpha \beta} \equiv \int_{0}^{\infty} d E \mathcal{T}_{E}^{\alpha \beta} u_{E}^{\beta} / h \geq 0$ and $\quad \mathcal{D}_{\alpha \beta} \equiv \mathcal{F}_{\alpha}-\mathcal{F}_{\beta}$. Here, we used that, at vanishing magnetic field, the transmission coefficients obey $\mathcal{T}_{E}^{\alpha \beta}=\mathcal{T}_{E}^{\beta \alpha}$ as a consequence of time-reversal symmetry [22,25-27]. Next, we observe that, for any $x$, the second sum in (9) is non-negative if $0 \leq$ $\psi \leq 2$ [28]. Hence, under this condition, the quadratic form $A_{\alpha}$ is positive semidefinite, since the first sum in (9) is generally non-negative. Consequently, setting $\psi=2$ in (8) and taking the minimum with respect to $x$ yields 


$$
\sigma \varepsilon_{\alpha} \geq 2 k_{B}
$$

For systems where time-reversal symmetry is broken by means of an external magnetic field, the transmission coefficients $\mathcal{T}_{E, \mathbf{B}}^{\alpha \beta}$ are, in general, not symmetric with respect to $\alpha$ and $\beta$. However, they still fulfill the weaker constraint $\sum_{\beta} \mathcal{T}_{E, \mathbf{B}}^{\alpha \beta}=\sum_{\beta} \mathcal{T}_{E . \mathbf{B}}^{\beta \alpha}$, which follows from the volumepreserving property of the scattering map (2) $[12,22,24]$. Using this sum rule, the quadratic form (8) can be expressed as

$$
\begin{aligned}
A_{\alpha}= & \sum_{\beta \neq \alpha} \sum_{\gamma} \mathcal{V}_{\mathbf{B}}^{\beta \gamma}\left(e^{\mathcal{D}_{\beta \gamma}}-1-\mathcal{D}_{\beta \gamma}\right) \\
& +\sum_{\beta} \mathcal{V}_{\mathbf{B}}^{\alpha \beta}\left\{(1+2 \psi x)\left(e^{\mathcal{D}_{\alpha \beta}}-1\right)\right. \\
& \left.+\psi x^{2}\left(e^{\mathcal{D}_{\alpha \beta}}+1\right)-\mathcal{D}_{\alpha \beta}\right\},
\end{aligned}
$$

where $\mathcal{V}_{\mathbf{B}}^{\alpha \beta} \geq 0$ is defined analogous to $\mathcal{V}^{\alpha \beta}$ in (9). Minimizing the term inside the curly brackets shows that the second sum in (11) is non-negative for any $x$ if

$0 \leq \psi \leq \min _{y \in \mathbb{R}} \frac{\left(1-e^{y}+y e^{y}\right)\left(e^{y}+1\right)}{\left(e^{y}-1\right)^{2}} \equiv \psi^{*} \simeq 0.89612$.

Moreover, the first contribution in (11), which does not depend on $x$, is non-negative due to the convexity of the exponential function. Hence, by using the same argument as in the derivation of (10), we arrive at

$$
\sigma \varepsilon_{\alpha} \geq \psi^{*} k_{B} .
$$

The bounds (10) and (13) constitute our first main result. Following from elementary microscopic principles, respectively, time-reversal symmetry and the conservation of phase-space volume, they hold for any scattering potential, any number of terminals, and arbitrarily far from equilibrium. On the macroscopic level, they imply that any increase in the precision $1 / \varepsilon_{\alpha}$, at which particles are extracted from the reservoir $\alpha$, inevitably leads to a proportional increase of the minimal thermodynamic cost $\sigma$ of the transport process. The symmetric bound (10) thereby has exactly the same form as the recently discovered thermodynamic uncertainty relation [1]. Indeed, (10) can also be obtained by mapping the classical scattering formalism to an effective Markov jump process. However, the local detailed-balance condition, which is crucial to the proof given in Refs. [2,3], is thereby fulfilled only for systems symmetric under time reversal [22]. Remarkably, (13) shows that the minimal cost of precision is reduced by a factor of $\psi^{*} / 2$ if this symmetry is broken by an external magnetic field.

To show that our bounds are tight, we consider an $\mathrm{N}$ terminal conductor with a flat target potential. An external magnetic field $\mathbf{B}$ forces incoming particles with mass $m$ and charge $q$ on bouncing orbits along the boundary of the target region (Fig. 2). This scattering mechanism is captured by the transmission coefficients

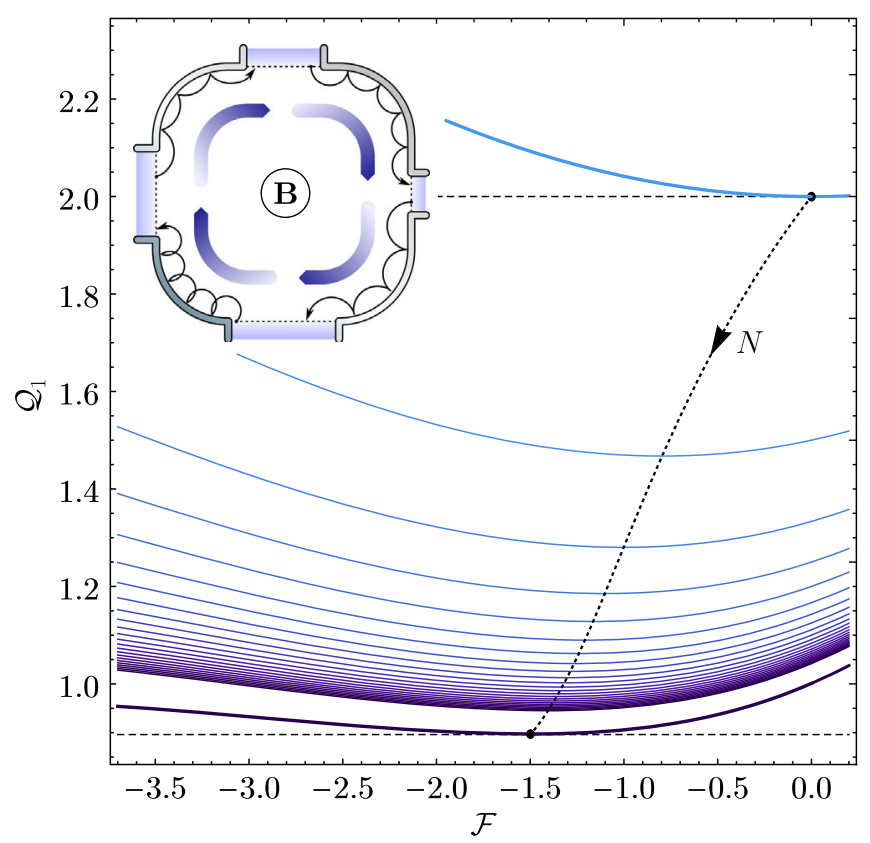

FIG. 2. Chiral transport. Bouncing orbits enforced by a strong magnetic field B sustain clockwise-oriented currents between adjacent leads (inset). From top to bottom, the blue blue lines show the cost-precision ratio $\mathcal{Q}_{1}$ defined in (15) as a function of the rescaled bias increment $\mathcal{F}$ for systems with $N=2, \ldots, 25$ terminals and for the limiting case $N \rightarrow \infty$. Interpolating between the minima of $\mathcal{Q}_{1}$, the dotted line crosses the two dashed lines, respectively indicating the bounds (10) and (13), at $N=2$ and $N \rightarrow \infty$.

$\mathcal{T}_{E, \mathbf{B}}^{\alpha \beta}=\sqrt{2 m E}\left\{\pi R_{E, \mathbf{B}} \delta_{\alpha \beta+1}+\left(2 l_{\alpha}-\pi R_{E, \mathbf{B}}\right) \delta_{\alpha \beta}\right\}$

with periodic indices $\alpha, \beta=1, \ldots, N$ [12]. The direction of the magnetic field has been chosen such that the Larmor circles with radius $R_{E, \mathbf{B}} \equiv \sqrt{2 m E} /(q|\mathbf{B}|)$ are oriented counterclockwise.

For a strong magnetic field, the typical Larmor radii are small compared to the dimensions of the conductor. Under this condition, the transmission coefficients are given by (14) throughout the relevant range of energies. Because of the asymmetric structure of these coefficients, a chiral steady state emerges, where currents flow in the clockwise direction between neighboring reservoirs [20]. To generate a net transfer of particles, an external bias has to be applied, breaking the $\mathrm{N}$-fold rotational symmetry of the system. For simplicity, here we choose the chemical potentials of the reservoirs to increase linearly in steps proportional to $1 / N$; that is, we set $\mathcal{F}_{\alpha} \equiv \alpha \mathcal{F} / N$. The mean currents and fluctuations can then be evaluated explicitly by inserting (14) into (4). Using the abbreviation $\mathcal{E} \equiv \exp [\mathcal{F} / N]$, we thus obtain the expressions

$$
\begin{aligned}
& \mathcal{Q}_{1}=\frac{\mathcal{F}}{N}\left(1+(N-1) \mathcal{E}^{N}-\frac{\mathcal{E}^{N}-1}{\mathcal{E}-1}\right) \frac{\mathcal{E}+\mathcal{E}^{N}}{\left(\mathcal{E}-\mathcal{E}^{N}\right)^{2}}, \\
& \mathcal{Q}_{\alpha}=\frac{\mathcal{F}}{N}\left(1+(N-1) \mathcal{E}^{N}-\frac{\mathcal{E}^{N}-1}{\mathcal{E}-1}\right) \frac{\mathcal{E}^{2}+\mathcal{E}}{\mathcal{E}^{\alpha}(\mathcal{E}-1)^{2}}(\alpha>1)
\end{aligned}
$$


for the dimensionless product $\mathcal{Q}_{\alpha} \equiv \sigma \varepsilon_{\alpha} / k_{B}$ of total dissipation (6) and relative uncertainty (7).

In the simplest case $N=2$, the transmission coefficients (14) are still symmetric, and (15) reduces to

$$
\mathcal{Q}_{\alpha}=\mathcal{F} \operatorname{coth}[\mathcal{F} / 4] / 2=2+\mathcal{F}^{2} / 24+\mathcal{O}\left(\mathcal{F}^{4}\right) .
$$

Hence, $\mathcal{Q}_{\alpha}$ reaches its minimum at $\mathcal{F}=0$, and the bound (10) is saturated in the linear response regime. As $N$ increases, the minimum of $\mathcal{Q}_{1}$ becomes successively smaller and shifts to negative values of $\mathcal{F}$ (Fig. 2). For large $N$, we obtain the asymptotic expression

$$
\left.\lim _{N \rightarrow \infty} \mathcal{Q}_{1}\right|_{\mathcal{F}<0}=\frac{\left(1-e^{\mathcal{F}}+\mathcal{F} e^{\mathcal{F}}\right)\left(e^{\mathcal{F}}+1\right)}{\left(e^{\mathcal{F}}-1\right)^{2}},
$$

which should be compared with (12). In fact, (17) reaches its minimal value $\psi^{*}$ at $\mathcal{F} \simeq-1.49888$. This result shows that the cost-precision ratio $\mathcal{Q}_{1}$ can come arbitrarily close to its lower bound (13) as the number of terminals increases. By contrast, $\mathcal{Q}_{\alpha>1}$ asymptotically grows as $N^{2}$ at any $\mathcal{F} \neq 0$. This divergence is a consequence of the chiral transmission coefficients (14) enabling the exchange of particles only between clockwise-adjacent reservoirs: The currents $J_{\alpha>1}$ are effectively driven by the bias $\mathcal{F} / N$ and hence vanish as $1 / N$, while the fluctuations $S_{\alpha>1}$ and the total dissipation $\sigma$ stay finite for large $N$.

So far, we have shown that precision in classical ballistic transport requires a minimal thermodynamic cost, which can be substantially reduced in systems with broken timereversal symmetry. Although our derivations were performed in a two-dimensional setting, it is straightforward to establish (10) and (13) also in one and three dimensions. Rather than spelling out the details of this procedure, in the last part of this Letter, we develop a perspective beyond the classical regime.

For a quantum theory of ballistic transport, the phasespace variable $J_{\alpha}\left[\xi_{t}\right]$ in (1) has to be promoted to an operator in the Heisenberg picture. Replacing classical trajectories with quantum states, the ensemble average in (1) can then be evaluated using standard techniques from quantum scattering theory [30,31]. In this formalism, the crucial role of the scattering map (2) is played by the complex scattering matrices $\mathbf{S}_{E, \mathbf{B}}^{\alpha \beta}$, which connect the amplitudes of incoming waves in the lead $\beta$ and outgoing waves in the lead $\alpha$, respectively [32]. For fermionic particles, the mean current is thus obtained as

$$
J_{\alpha}=\frac{1}{h} \int_{0}^{\infty} d E \sum_{\beta} \hat{\mathcal{T}}_{E, \mathbf{B}}^{\alpha \beta}\left(f_{E}^{\alpha}-f_{E}^{\beta}\right) .
$$

Notably, this expression has the same structure as its classical correspondent (4) with the quantum transmission coefficients defined as

$$
\hat{\mathcal{T}}_{E, \mathbf{B}}^{\alpha \beta} \equiv 2 \operatorname{tr}\left[\mathbf{T}_{E, \mathbf{B}}^{\alpha \beta}\right] \quad \text { with } \quad \mathbf{T}_{E, \mathbf{B}}^{\alpha \beta} \equiv \mathbf{S}_{E, \mathbf{B}}^{\alpha \beta}\left(\mathbf{S}_{E, \mathbf{B}}^{\alpha \beta}\right)^{\dagger}
$$

and the Maxwell-Boltzmann distribution (5) replaced by the Fermi-Dirac distribution

$$
f_{E}^{\alpha} \equiv 1 /\left\{1+\exp \left[\left(E-\mu_{\alpha}\right) /\left(k_{B} T\right)\right]\right\} .
$$

The anatomy of current fluctuations in the quantum regime is, however, more complicated than in the classical case; $S_{\alpha}=S_{\alpha}^{\mathrm{cl}}-S_{\alpha}^{\mathrm{qu}}$ involves two components [32]:

$$
\begin{aligned}
S_{\alpha}^{\mathrm{cl}} & \equiv \frac{1}{h} \int_{0}^{\infty} d E \sum_{\beta \neq \alpha} \hat{\mathcal{T}}_{E, \mathbf{B}}^{\alpha \beta}\left\{f_{E}^{\alpha}\left(1-f_{E}^{\beta}\right)+f_{E}^{\beta}\left(1-f_{E}^{\alpha}\right)\right\}, \\
S_{\alpha}^{\mathrm{qu}} & \equiv \frac{2}{h} \int_{0}^{\infty} d E \sum_{\beta \gamma} \operatorname{tr}\left[\mathbf{T}_{E, \mathbf{B}}^{\alpha \beta} \mathbf{T}_{E, \mathbf{B}}^{\alpha \gamma}\right]\left(f_{E}^{\alpha}-f_{E}^{\beta}\right)\left(f_{E}^{\alpha}-f_{E}^{\gamma}\right),
\end{aligned}
$$

both of which are non-negative. Depending only on singleparticle quantities, $S_{\alpha}^{\mathrm{cl}}$ can be regarded as the quantum analogue of the classical expression (4) with additional Pauli-blocking factors accounting for the exclusion principle. By contrast, the contribution $S_{\alpha}^{\mathrm{qu}}$, which is of second order in the transmission matrices $\mathbf{T}_{E, \mathbf{B}}^{\alpha \beta}$ and hence describes the correlated exchange of two particles, has no classical counterpart [32].

The two-component structure (21) of the current fluctuations suggests to divide the relative uncertainty $\varepsilon_{\alpha}=$ $\varepsilon_{\alpha}^{\mathrm{cl}}-\varepsilon_{\alpha}^{\mathrm{qu}}$ into a quasiclassical part $\varepsilon_{\alpha}^{\mathrm{cl}} \equiv S_{\alpha}^{\mathrm{cl}} / J_{\alpha}^{2}$ and a quantum correction $\varepsilon_{\alpha}^{\mathrm{qu}} \equiv S_{\alpha}^{\mathrm{qu}} / J_{\alpha}^{2}$. By following the lines leading to (10) and (13), it is then possible to establish the bounds [22,33]

$$
\sigma \varepsilon_{\alpha}^{\mathrm{cl}} \geq 2 k_{B} \quad \text { and } \quad \sigma \varepsilon_{\alpha}^{\mathrm{cl}} \geq \psi^{*} k_{B},
$$

respectively, for quantum systems with and without timereversal symmetry, where $\sigma=k_{B} \sum_{\alpha} \mathcal{F}_{\alpha} J_{\alpha}$. As in the classical case, this result follows from the symmetry $\hat{\mathcal{T}}_{E}^{\alpha \beta}=$ $\hat{\mathcal{T}}_{E}^{\beta \alpha}$ of the quantum transmission coefficients (19) for $\mathbf{B}=0$ and from the sum rules $\sum_{\beta} \hat{\mathcal{T}}_{E, \mathbf{B}}^{\alpha \beta}=\sum_{\beta} \hat{\mathcal{T}}_{E, \mathbf{B}}^{\beta \alpha}$ for $\mathbf{B} \neq 0$ [32]. It implies, in particular, that the classical relations (10) and (13) are recovered close to equilibrium, i.e., for small affinities $\mathcal{F}_{\alpha}$, and in the semiclassical regime, where the fugacities $\varphi_{\alpha} \equiv \exp \left[\mu_{\alpha} /\left(k_{B} T\right)\right]$ are small [27]; in both cases, the quantum fluctuations $S_{\alpha}^{\mathrm{qu}}$ are negligible.

In general, however, the quantum corrections $\varepsilon_{\alpha}^{\mathrm{qu}}$ will spoil the bounds (10) and (13) as the following simple model shows. Consider a two-terminal conductor with narrow leads allowing only for a single open transport channel; i.e., the system is effectively one dimensional and the scattering matrices $\mathbf{S}_{E, \mathbf{B}}^{\alpha \beta}$ reduce to complex numbers. The target acts as a perfect energy filter, which is fully transparent in a small window $\Delta$ around the reference chemical potential $\mu$ and opaque at all other energies. Such filters are standard tools in mesoscopic physics [34-36] and can be implemented, for example, with quantum Hall edge states [37]. Setting $\mathcal{F}_{1} \equiv-\mathcal{F}_{2} \equiv \mathcal{F} / 2$ and neglecting second-order corrections in $\Delta /\left(k_{B} T\right)$, we obtain 
$\sigma \varepsilon_{\alpha}^{\mathrm{cl}}=k_{B} \mathcal{F} \operatorname{coth}[\mathcal{F} / 2] \quad$ and $\quad \sigma \varepsilon_{\alpha}=k_{B} \mathcal{F} / \sinh [\mathcal{F} / 2]$

by evaluating (18) and (21) [22]. Hence, while the product of total dissipation $\sigma$ and quasiclassical uncertainty $\varepsilon_{\alpha}^{\mathrm{cl}}$ is bounded by $2 k_{B}$, the full cost-precision ratio $\sigma \varepsilon_{\alpha}$ can become arbitrarily small. Specifically, as $\mathcal{F}$ becomes large, the current $J_{\alpha}$ saturates to a finite value, $\sigma$ grows linearly and the current noise $S_{\alpha}$ decays exponentially. This behavior can be understood from the fact that, in fermionic systems, fluctuations in the occupation of individual scattering states are strongly suppressed at a large bias; the scattering states emerging from the high-bias reservoir are filled almost completely, while the ones going in the opposite direction are essentially empty.

The example above shows that a combination of quantum effects and energy filtering makes it possible to exponentially reduce the minimal thermodynamic cost of precision. Whether or not this phenomenon can be captured in a generalized trade-off relation, where either cost or precision enters nonlinearly, remains an intriguing question for future research. Further prospects include the extension of our theory to systems with temperature gradients or bosonic particles.

Notably, the number $\psi^{*}$, which enters the nonsymmetric bounds (13) and (22), also appears in a recently found tradeoff relation between the power and efficiency of stochastic heat engines [29]. These figures are indeed connected with the minimal cost of precision [38]. Using our approach, it might thus be possible to bound the performance of ballistic thermoelectric engines, a class of devices that is currently subject to active investigations; see, for example, $[12,13,16-$ 20,34-37,39]. At this point, we conclude by stressing that any violation of our classical bounds constitutes a clear signature of quantum effects. Therefore, our work provides a valuable new benchmark to probe nonclassical transport mechanisms in future theoretical and experimental studies.

K. B. acknowledges financial support from the Academy of Finland (Contract No. 296073) and is affiliated with the Centre of Quantum Engineering. K. B. thanks P. Pietzonka, P. Burset, K. Macieszczak, and M. Moskalets for insightful discussions and U. Seifert for long-standing support. K. S. was supported by JSPS Grants-in-Aid for Scientific Research (No. JP25103003, No. JP16H02211, and No. JP17K05587). This research was supported in part by the International Centre for Theoretical Sciences (ICTS) during a visit for participating in the program-Large deviation theory in statistical physics: Recent advances and future challenges (Code: ICTS/Prog-ldt/2017/8).

[1] A. C. Barato and U. Seifert, Thermodynamic Uncertainty Relation for Biomolecular Processes, Phys. Rev. Lett. 114, 158101 (2015).

[2] T. R. Gingrich, J. M. Horowitz, N. Perunov, and J. L. England, Dissipation Bounds All Steady-State Current Fluctuations, Phys. Rev. Lett. 116, 120601 (2016).
[3] T. R. Gingrich, G. M. Rotskoff, and J. M. Horowitz, Inferring dissipation from current fluctuations, J. Phys. A 50, 184004 (2017).

[4] P. Pietzonka, F. Ritort, and U. Seifert, Finite-time generalization of the thermodynamic uncertainty relation, Phys. Rev. E 96, 012101 (2017).

[5] J. M. Horowitz and T. R. Gingrich, Proof of the finite-time thermodynamic uncertainty relation for steady-state currents, Phys. Rev. E 96, 020103(R) (2017).

[6] K. Proesmans and C. Van den Broeck, Discrete-time thermodynamic uncertainty relation, Europhys. Lett. 119, 20001 (2017).

[7] A. C. Barato and U. Seifert, Cost and Precision of Brownian Clocks, Phys. Rev. X 6, 041053 (2016).

[8] C. Hyeon and W. Hwang, Physical insight into the thermodynamic uncertainty relation using Brownian motion in tilted periodic potentials, Phys. Rev. E 96, 012156 (2017).

[9] A. Dechant and S. Sasa, Current fluctuations and transport efficiency for general Langevin systems, arXiv:1708.08653.

[10] T. E. Humphrey, R. Newbury, R. P. Taylor, and H. Linke, Reversible Quantum Brownian Heat Engines for Electrons, Phys. Rev. Lett. 89, 116801 (2002).

[11] T. E. Humphrey and H. Linke, Reversible Thermoelectric Nanomaterials, Phys. Rev. Lett. 94, 096601 (2005).

[12] J. Stark, K. Brandner, K. Saito, and U. Seifert, Classical Nernst Engine, Phys. Rev. Lett. 112, 140601 (2014).

[13] M. Horvat, T. Prosen, and G. Casati, Exactly solvable model of a highly efficient thermoelectric engine, Phys. Rev. E 80, 010102(R) (2009).

[14] G. Casati, C. Mejía-Monasterio, and T. Prosen, Increasing Thermoelectric Efficiency: A Dynamical Systems Approach, Phys. Rev. Lett. 101, 016601 (2008).

[15] G. Casati, C. Mejía-Monasterio, and T. Prosen, Magnetically Induced Thermal Rectification, Phys. Rev. Lett. 98, 104302 (2007).

[16] K. Brandner, K. Saito, and U. Seifert, Strong Bounds on Onsager Coefficients and Efficiency for Three-Terminal Thermoelectric Transport in a Magnetic Field, Phys. Rev. Lett. 110, 070603 (2013).

[17] K. Brandner and U. Seifert, Multi-terminal thermoelectric transport in a magnetic field: Bounds on Onsager coefficients and efficiency, New J. Phys. 15, 105003 (2013).

[18] B. Sothmann, R. Sánchez, and A. N. Jordan, Quantum Nernst engines, Europhys. Lett. 107, 47003 (2014).

[19] K. Brandner and U. Seifert, Bound on thermoelectric power in a magnetic field within linear response, Phys. Rev. E 91, 012121 (2015).

[20] R. Sánchez, B. Sothmann, and A. N. Jordan, Chiral Thermoelectrics with Quantum Hall Edge States, Phys. Rev. Lett. 114, 146801 (2015).

[21] E. Ott, Chaos in Dynamical Systems, 2nd ed. (Cambridge University Press, Cambridge, England, 2009).

[22] See Supplemental Material at http://link.aps.org/ supplemental/10.1103/PhysRevLett.120.090601 for a brief review of the classical scattering formalism and derivations of the results (22) and (23).

[23] U. Seifert, Stochastic thermodynamics, fluctuation theorems and molecular machines, Rep. Prog. Phys. 75, 126001 (2012). 
[24] J. Stark, Master thesis, University of Stuttgart, 2013.

[25] L. Onsager, Reciprocal Relations in Irreversible Processes I, Phys. Rev. 37, 405 (1931).

[26] H. B. G. Casimir, On Onsager's principle of microscopic reversibility, Rev. Mod. Phys. 17, 343 (1945).

[27] H. B. Callen, Thermodynamics and an Introduction to Thermostatics, 2nd ed. (Wiley, New York, 1985).

[28] This bound can be verified by minimizing the term inside the curly brackets in (9) with respect to $x$ and using that $y\left(e^{y}-1\right) \geq 2\left(e^{y}-1\right)^{2} /\left(e^{y}+1\right)$ for any $y \in \mathbb{R}[29]$.

[29] N. Shiraishi, K. Saito, and H. Tasaki, Universal Trade-Off Relation between Power and Efficiency for Heat Engines, Phys. Rev. Lett. 117, 190601 (2016).

[30] P. Gaspard, Multivariate fluctuation relations for currents, New J. Phys. 15, 115014 (2013).

[31] P. Gaspard, Scattering theory and thermodynamics of quantum transport, Ann. Phys. (Amsterdam) 527, 663 (2015).

[32] Y. V. Nazarov and Y. M. Blanter, Quantum TransportIntroduction to Nanoscience, 1st ed. (Cambridge University Press, Cambridge, England, 2009).
[33] Gheorghe Nenciu, Independent electron model for open quantum systems: Landauer-Buttiker formula and strict positivity of the entropy production, J. Math. Phys. (N.Y.) 48, 033302 (2007).

[34] K. Yamamoto and N. Hatano, Thermodynamics of the mesoscopic thermoelectric heat engine beyond the linearresponse regime, Phys. Rev. E 92, 042165 (2015).

[35] R. S. Whitney, Most Efficient Quantum Thermoelectric at Finite Power Output, Phys. Rev. Lett. 112, 130601 (2014).

[36] G. Benenti, G. Casati, K. Saito, and R. S. Whitney, Fundamental aspects of steady-state conversion of heat to work at the nanoscale, Phys. Rep. 694, 1 (2017).

[37] P. Samuelsson, S. Kheradsoud, and B. Sothmann, Optimal Quantum Interference Thermoelectric Heat Engine with Edge States, Phys. Rev. Lett. 118, 256801 (2017).

[38] P. Pietzonka and U. Seifert, Universal trade-off between power, efficiency and constancy in steady-state heat engines, arXiv:1705.05817v1.

[39] K. Saito, G. Benenti, and G. Casati, A microscopic mechanism for increasing thermoelectric efficiency, Chem. Phys. 375, 508 (2010). 